\title{
Bone Safety of Dolutegravir-Containing Regimens in People Living with HIV: Results from a Real-World Cohort
}

This article was published in the following Dove Press journal: Infection and Drug Resistance

\author{
Paolo Bonfanti $\mathbb{D}^{\prime}$ \\ Andrea De Vito $\mathbb{D}^{2}$ \\ Elena Ricci (iD ${ }^{3}$ \\ Barbara Menzaghi ${ }^{4}$ \\ Giancarlo Orofino (iD ${ }^{5}$ \\ Nicola Squillace (D) ${ }^{\prime}$ \\ Chiara Molteni ${ }^{6}$ \\ Giuseppe Vittorio De Socio (iD) ${ }^{7}$ \\ Elena Salomoni (iD) ${ }^{8}$ \\ Benedetto Maurizio Celesia (iD ${ }^{9}$ \\ Chiara Dentone (iD) ${ }^{10}$ \\ Valeria Colombo iD "I \\ Giordano Madeddu (iD ${ }^{2}$ \\ 'Infectious Diseases Unit ASST-MONZA, San \\ Gerardo Hospital-University of Milano-Bicocca, \\ Milan, Italy; ${ }^{2}$ Unit of Infectious Diseases, \\ Department of Medical, Surgical and \\ Experimental Sciences, University of Sassari, \\ Sassari, Italy; ${ }^{3}$ Fondazione IRCCS Ca' Granda \\ Ospedale Maggiore Policlinico, Milan, Italy; \\ ${ }^{4}$ Unit of Infectious Diseases, ASST della Valle \\ Olona - Busto Arsizio (VA), Busto Arsizio, \\ Italy; ${ }^{5}$ Division I of Infectious and Tropical \\ Diseases, ASL Città di Torino, Turin, Italy; ' $U n i t$ \\ of Infectious Diseases, A. Manzoni Hospital, \\ Lecco, Italy; ${ }^{7}$ Department of Internal Medicine \\ 2, Infectious Diseases Unit, Perugia "Santa \\ Maria della Misericordia" General Hospital, \\ Perugia, Italy; ${ }^{8}$ Infectious Diseases Unit I, Santa \\ Maria Annunziata Hospital, Azienda USL \\ Toscana Centro, Florence, Italy; ${ }^{9}$ Infectious \\ Diseases University of Catania ARNAS \\ Garibaldi Catania, Catania, Italy, ${ }^{10}$ Division of \\ Infectious Diseases, Department of Health \\ Sciences, IRCCS Ospedale Policlinico San \\ Martino, Genova, Italy; "Infectious Disease \\ Unit, DIBC "Luigi Sacco", University of Milan, \\ Milan, Italy
}

Correspondence: Giordano Madeddu Unit of Infectious Diseases, Department of Medical, Surgical and Experimental

Sciences, University of Sassari, Viale San

Pietro 43, Sassari 07100, Italy

Tel +393403781734

Fax +39079217620

Email giordano@uniss.it
Objective: Few data exist about the effect of dolutegravir (DTG) on bone mineral density (BMD) in real life. The aim of this study was to determine rates of change in BMD over time in people living with HIV (PLWH) treated with DTG.

Design: The SCOLTA project is a multicenter observational study enrolling HIV-infected people who start newly commercialized drugs prospectively, with the aim of identifying toxicities and adverse events (AE) in a real-life setting.

Methods: Dual-energy X-ray absorptiometry at the femoral neck (FN) and lumbar spine (LS) was performed at study entry (baseline, BL) and after 96 weeks. Percentage BMD change from BL was evaluated using a general linear model, including factors potentially associated with bone loss.

Results: One hundred and sixty PLWH were enrolled (26.3\% female, mean age $49.9 \pm 11.2$ years) from April 2015 to April 2017. Overall, we could calculate BMD change from baseline, for at least one site, in 133 subjects (83.1\%). After a median of 102 weeks (IQR: 90-110), mean FN BMD increased, but not significantly, whereas LS BMD showed a significant mean increase of 13.1 (95\% confidence interval, CI: $1.7-24.6) \mathrm{mg} / \mathrm{cm}^{3}(+1.6 \%, 95 \%$ CI: $0.3 \%, 2.8 \%)$ after a median time of 102 weeks (IQR: 84-110). As regards LS BMD, patients with osteopenia/ osteoporosis at study entry experienced a high increase from baseline (20.6, 95\% CI: 3.1, $\left.38.1 \mathrm{mg} / \mathrm{cm}^{3}\right)$, as well as experienced subjects $\left(16.9,95 \% \mathrm{CI}: 4.7,29.2 \mathrm{mg} / \mathrm{cm}^{3}\right)$ and those on vitamin D supplementation (26.8, 95\% CI: $\left.7.7,45.9 \mathrm{mg} / \mathrm{cm}^{3}\right)$.

Conclusion: Dolutegravir-containing regimens could reduce the negative impact of antiretroviral therapy on bone, especially in patients with low BMD.

Keywords: HIV infection, dolutegravir, bone mineral density, real-life setting, adverse events, DXA scan

\section{Introduction}

Following the introduction of combination antiretroviral therapy (cART), life expectancy lengthened, and HIV infection became a chronic condition. As a result, several HIV-associated non-AIDS comorbidities appeared, including bone disease such as osteoporosis and increase the risk of fracture. ${ }^{1-3}$ As a matter of fact, studies reported osteopenia in up to two-thirds, and osteoporosis in about $15 \%$, of HIV-infected patients. ${ }^{4,5}$

People living with HIV (PLWH) have both traditional (low body mass index (BMI), previous fracture, use of tobacco, alcohol, illicit drugs, etc.) and HIVspecific risk factors. These include antiretroviral drugs, chronic inflammation, 
immune reconstitution, ${ }^{6}$ hepatitis $\mathrm{C}$ virus $(\mathrm{HCV})$ or hepatitis $\mathrm{B}$ virus (HBV), co-infections, diabetes, and renal disease. ${ }^{7}$ Furthermore, in PLWH, vitamin D deficiency is more common than in general population ${ }^{8,9}$ due to the effect of antiretroviral drugs (especially efavirenz and protease inhibitors), ${ }^{8,10,11}$ and HIV-related immuneactivation. $^{12}$

cART initiation is associated with approximately $6 \%$ bone loss in the first two years of treatment. ${ }^{5}$ After that, bone mineral density (BMD) may stabilize, increase, or further decrease, depending on the drug regimen. ${ }^{7,13}$

Several studies showed that patients treated with tenofovir disoproxil fumarate (TDF) suffered a greater reduction in bone mineral density than those treated with other antiretroviral drugs. ${ }^{4,8,14,15}$ This reduction was not observed in patients who started tenofovir alafenamide (TAF)-including cART. ${ }^{16-18}$ Furthermore, raltegravircontaining regimens were associated with inferior loss or even with BMD increase over time. .,19,20 $^{-1}$

Dolutegravir (DTG) is a second-generation integrase inhibitor (INSTI) that has shown to be effective and safe in both naïve, including late (CD4 count $<350$ cells $/ \mathrm{mm}^{3}$ ) and AIDS-presenters, and experienced patients. ${ }^{21,22}$ DTG has also been used with switch strategies to improve lipid profiles in experienced patients. ${ }^{23-25}$

To date, few studies reported on the impact of DTG on bone health. ${ }^{26-28}$ In the framework of a surveillance cohort on adverse events of antiretroviral drugs, we aimed to determine rates of change in BMD over time in PLWH treated with DTG in both naïve and experienced PLWH. We also investigated other potential risk factors that could impact BMD changes.

\section{Methods}

The SCOLTA Project (Surveillance Cohort Long-Term Toxicity of Antiretrovirals) is a prospective, observational, multicenter study created to assess the incidence of adverse events (AEs) in patients receiving new antiretroviral drugs in clinical practice. The study protocol was approved by the local ethics committee of the coordinating center at Hospital "L. Sacco"-University of Milan on 18 September 2002. A new protocol amendment was submitted and approved on 13 June 2013 by the same ethics committee. This last version was approved by the local ethics committee of each group, and written consent was obtained from all participants.

The SCOLTA Project is an on-line pharmacovigilance program involving 25 Italian Infectious Disease Departments. The Project has an internet site (http:// www.cisai.it) where grades 3 and 4 AEs are recorded (http://rcc.tech-res-intl.com/tox tables.htm).

Patients who start taking a cohort drug are consecutively proposed to enter that cohort and asked to give written informed consent. As this is an observational study, the choice of therapy is entirely up to the individual physicians and patients in each center. Patients are followed up according to the standards of each center. At baseline, clinical characteristics, laboratory variables, ART history, and comorbidities are recorded.

Patients undergo follow-up at 6-month intervals, and AEs are notified when they are clinically observed. AEs are described according to the Division of AIDS table. ${ }^{29}$ Complete data collection and follow-up procedures for the cohorts are described elsewhere. ${ }^{30,31}$ We defined "dual therapy" as DTG plus another antiretroviral drug. Virological failure or discontinuation was defined by the clinicians according to current national guidelines. ${ }^{32}$ The estimated glomerular filtration rate (eGFR) was calculated using the Modification of Diet in Renal Diseases (MDRD) formula. The SCOLTA Project currently includes two cohorts: bictegravir and dolutegravir.

Out of 25 participating centers, 10 perform dual-energy X-ray absorptiometry (DXA) scan in their outpatient clinics as a part of a routine follow-up visit. Some centers performed both lumbar spine (LS), and femoral neck (FN) scans, others just at one site (LS or FN). To investigate the bone safety of dolutegravir, we proposed the study to patients who had a DXA scan performed in the two months before or after DTG initiation and referred to a center that could internally carry out a control DXA during the 2-year follow-up period. Individuals receiving ongoing treatment for bone disease (eg, osteoporosis), including bisphosphonates, denosumab, and strontium ranelate or those receiving chronic treatment with drugs that could affect bone metabolism (glucocorticoids) were excluded from this analysis.

DXA was performed using Lunar or Hologic. We transformed Lunar results with the equations provided by Hologic. ${ }^{33}$ T-score and Z-score were calculated using the Italian standard of the Densitometric Italian Normative Study $^{34}$ as the reference and osteopenia and osteoporosis were defined as per WHO definition ${ }^{35}$ if T-score was lower than -1.0 and -2.5 , respectively, at one site at least.

\section{Statistical Analysis}

Data were described using mean ( \pm standard deviation, SD) for normally distributed continuous variables, median 
(interquartile range, IQR) for not normally distributed continuous variables, frequency (\%) for categorical and ordinal variables. Changes from baseline were described as means and 95\% confidence intervals (CI) and analyzed using a T-student test for paired data to ascertain if they were significantly different from 0 . Mean changes from baseline were compared between groups using the analysis of variance.

A general linear model was used to include potential confounders in the multivariate analysis. Changes from baseline of BMD (FN and LS, both absolute and percentage) were evaluated including factors associated with different BMD at baseline or affecting BMD modification during follow-up, that is age, sex, BMI, osteopenia/osteoporosis at baseline, vitamin D supplementation, weeks to control scan, and being ART naïve or experienced at baseline. The multivariate analysis was also run in ART experienced group, including previous exposure to PI or TDF. Adjusted mean changes from baseline were deemed significant if 95\% CI excluded the zero, and between-group differences if $\mathrm{P}$ from the multivariable model was $<0.05$.

All patients with at least one follow-up BMD measure were included in the intention-to-treat (ITT) analysis. To evaluate if the results were significantly different, we planned to perform a sensitivity analysis excluding patients who interrupted DTG before undergoing the follow-up DXA scan.

We did not perform any adjustment for multiple testing, given the exploratory nature of this study and the limited sample size.

Statistical analysis was performed using the SAS/STAT statistical package (version 9.4; SAS Institute Inc., Cary, North Carolina, USA).

\section{Results}

Out of all patients enrolled in the DTG cohort from April 2015 until February 2017, 160 met the entry criteria. The last patient underwent the DXA follow-up scan in April 2019.

\section{Baseline Characteristics}

Comparing patients enrolled in the participating and nonparticipating centers over the study period, we did not find differences in terms of age, sex, BMI, CDC stage C, CD4 count and undetectable HIV-RNA (Table 1S). On the contrary, patients in participating centers were more frequently naïve, acquired HIV infection by sexual route, and had protease inhibitor (PI) in the previous regimen. Moreover, they had osteopenia or osteoporosis in significantly higher proportion, although this may reflect the center organization, in that DXA scan could be more easily performed in their outpatient clinics, leading to a higher proportion of diagnosis.

In the participating centers, no significant difference was observed between patients who did and did not undergo a DXA scan, with the exception of a high proportion of Vitamin D supplementation in those who had the scan (Table 2S).

The baseline characteristics of 160 patients included in this analysis are reported in Table 1 . There were no differences in age between women and men $(51.7 \pm 10.6$ vs 49.3 \pm 11.4 years, $\mathrm{p}=0.22$ ), whereas the former were less frequently naïve $(9.5 \%$ vs $28.0 \%, \mathrm{p}=0.015)$. The proportion of PLWH aged $\geq 50$ years was $61.9 \%$ in females and $55.1 \%$ in males $(p=0.44)$. Overall, 37 (23.1\%) PLWH were antiretroviral therapy naive. Among patients with known smoking status $(72.5 \%)$, current smokers were $35.4 \%$ and former smokers $12.1 \%$. Current smoking was more frequent in people aged $<50$ and former smoking in those $\geq 50$, but this trend was not significant.

At enrolment, $25(15.6 \%)$ subjects already had a diagnosis of osteopenia or osteoporosis. However, after performing the DXA scan, we found that 87 patients (54.4\%) had osteopenia or osteoporosis at one site at least; $48.1 \%$ of patients had lower BMD at LS and $24.4 \%$ at FN. One hundred thirty-eight (86.3\%) underwent a Hologic and 22 (13.7\%) a Lunar scan.

Mean BMD at baseline was $822 \pm 166 \mathrm{mg} / \mathrm{cm}^{3}$ at $\mathrm{FN}$ and $935 \pm 167 \mathrm{mg} / \mathrm{cm}^{3}$ at LS. According to selected characteristics, BMD means are reported in Table 2.

\section{Follow-Up Results}

Among 133 patients with baseline FN DXA scan, 112 (84.2\%) also had a follow-up scan after a median of 102 weeks (IQR: 90-110); among 157 patients with baseline LS scan, 128 had a control (81.5\%) after a median time of 102 weeks (IQR: 84-110). Overall, we could calculate BMD change from baseline, for at least one site, in 133 subjects (83.1\%). In 114 (85.7\%), BMD was measured by Hologic scan, and in $19(14.3 \%)$, a Lunar scan; the type of follow-up scan was different from baseline in three (Hologic to Lunar) and seven (Lunar to Hologic) subjects, respectively.

No significant difference was observed between the baseline characteristics of patients who did and did not perform a control DXA examination (Table 3S): the two groups were similar in most characteristics, although patients without follow-up scans were leaner and more frequently naïve. The reason for not undergoing followup DXA was the patient's choice. 
Table I Baseline Characteristics of the 160 Patients Enrolled in the Study

\begin{tabular}{|c|c|c|}
\hline \multicolumn{3}{|l|}{ Variables } \\
\hline $\operatorname{Sex}(n, \%)$ & & \\
\hline Female & 42 & $26.3 \%$ \\
\hline Male & 118 & $73.8 \%$ \\
\hline Age, years (mean, SD) & 49.9 & 11.2 \\
\hline BMI, $\mathbf{K g} / \mathbf{m}^{2}$ (mean, SD) & 24.0 & 4.2 \\
\hline BMI, $\mathrm{Kg} / \mathrm{m}^{2}$ (n, \%) & & \\
\hline$<25.0$ & 105 & $65.6 \%$ \\
\hline$\geq 25$ & 55 & $34.4 \%$ \\
\hline Risk factor for HIV acquisition (n, \%) & & \\
\hline Sexual route & 119 & $74.4 \%$ \\
\hline Intravenous drug use & 20 & $12.5 \%$ \\
\hline Other & 21 & $13.1 \%$ \\
\hline HCV Ab Positive (n, \%) & 24 & $15.0 \%$ \\
\hline Ethnicity (n, \%) & & \\
\hline Caucasians & 146 & $91.2 \%$ \\
\hline Other & 14 & $8.8 \%$ \\
\hline CDC stage (n, \%) & & \\
\hline A & 87 & $54.4 \%$ \\
\hline B & 38 & $23.8 \%$ \\
\hline C & 35 & $21.9 \%$ \\
\hline Naive (n, \%) & 37 & $23.1 \%$ \\
\hline HIV-RNA Undetectable (n, \%) (exp.pts) & 104 & $84.6 \%$ \\
\hline CD4 cells/mL (median, IQR) & 531 & $346-786$ \\
\hline CD8 cells/mL (median, IQR) & 795 & $606-1098$ \\
\hline CD4/CD8 (median, IQR) & 0.67 & $0.41-0.96$ \\
\hline Previous regimen including $(n, \%)$ & & \\
\hline Protease inhibitors & 48 & $30.0 \%$ \\
\hline Non nucleoside reverse transcriptase inhibitors & 51 & $31.9 \%$ \\
\hline Years of ART (median, IQR) & 9.7 & $4.0-18.0$ \\
\hline Current regimen including DTG (n, \%) & & \\
\hline Triple & 125 & $78.1 \%$ \\
\hline 3TC/ABC/DTG & 105 & $65.6 \%$ \\
\hline TDF/FTC/DTG & 20 & $12.5 \%$ \\
\hline Dual & 28 & $17.5 \%$ \\
\hline Other combinations & 7 & $4.4 \%$ \\
\hline $\begin{array}{l}\text { Previous diagnosis of osteopenia/ } \\
\text { osteoporosis (n, \%) }\end{array}$ & 25 & $15.6 \%$ \\
\hline $\begin{array}{l}\text { New diagnosis of osteopenia/osteoporosis } \\
(n, \%), n=135\end{array}$ & 62 & $45.9 \%$ \\
\hline Vitamin D supplementation (n, \%) & 52 & $32.5 \%$ \\
\hline Femoral neck $\mathrm{mg} / \mathrm{cm}^{3}$ (mean, SD) & 822 & 166 \\
\hline Lumbar spine $\mathrm{mg} / \mathrm{cm}^{3}$ (mean, SD) & 935 & 167 \\
\hline
\end{tabular}

Abbreviations: SD, standard deviation; IQR, interquartile range; BMI, body mass index; $\mathrm{HCV}$, hepatitis $\mathrm{C}$ virus; CDC, center for disease control and prevention; 3TC, lamivudine; ABC, abacavir; DTG, dolutegravir; TDF, tenofovir disoproxil fumarate; FTC, emtricitabine.
Table 2 Baseline Bone Mineral Density (BMD) According to Selected Characteristics in 160 Subjects with DXA at Femoral Neck and/or Lumbar Spine

\begin{tabular}{|l|l|l|l|l|}
\hline $\begin{array}{l}\text { Baseline } \\
\text { Characteristics }\end{array}$ & $\begin{array}{l}\text { FN BMD } \\
\left(\mathbf{m g} / \mathbf{c m}^{3}\right) \\
\text { Mean } \pm \text { SD }\end{array}$ & p-value* & $\begin{array}{l}\text { LS BMD } \\
\left(\mathbf{m g} / \mathbf{c m}^{3}\right) \\
\text { Mean } \pm \text { SD }\end{array}$ & p-value* \\
\hline Age $<50$ years & $861 \pm 175$ & 0.02 & $950 \pm 170$ & 0.30 \\
Age $\geq 50$ years & $793 \pm 154$ & & $923 \pm 164$ & \\
Men & $840 \pm 173$ & 0.048 & $956 \pm 170$ & 0.007 \\
Women & $776 \pm 139$ & & $875 \pm 140$ & \\
BMI<25.0 & $782 \pm 160$ & 0.0002 & $921 \pm 186$ & 0.14 \\
BMI $\geq 25.0$ & $891 \pm 155$ & & $963 \pm 117$ & \\
Experienced & $821 \pm 159$ & 0.92 & $923 \pm 156$ & 0.10 \\
Naive & $825 \pm 196$ & & $974 \pm 194$ & \\
\hline
\end{tabular}

Note: *Analysis of variance.

Abbreviations: BMD, bone mineral density; FN, femoral neck; SD, standard deviation; LS, lumbar spine; BMI, body mass index.

In Figure 1, mean BMD are shown for the entire sample at baseline and at follow-up. FN BMD increased in both absolute terms $\left(7.9,95 \% \mathrm{CI}:-6.3,22.2 \mathrm{mg} / \mathrm{cm}^{3}\right)$ and percentage $(1.4 \%, 95 \% \mathrm{CI}:-0.5 \%, 3.4 \%)$, but not significantly, whereas LS BMD showed a significant $(\mathrm{p}=0.025)$ mean increase of $13.1(95 \% \mathrm{CI}: 1.7-24.6) \mathrm{mg} /$ $\mathrm{cm}^{3}$ (1.6\%, 95\% CI: $\left.0.3 \%, 2.8 \%, \mathrm{p}=0.019\right)$.

To better understand if this bone mass density increase was more marked in some groups, we also performed univariate analyses of the change from baseline in strata of age ( $<$ and $\geq 50$ years), sex, BMI $(\leq 25.0$ and $>25.0$ ), vitamin $\mathrm{D}$ supplementation, naïve status, normal bone density at baseline, and according to PI or TDF in the last regimen (Table 3). As regards LS BMD, on average experienced subjects showed a significant positive modification $\left(+16.9,95 \%\right.$ CI: $4.7,29.2 \mathrm{mg} / \mathrm{cm}^{3}$, and $+2.0 \%, 95 \%$ CI: $0.6 \%, 2.4 \%$ ) whereas naive PLWH showed a slight and not significant decrease. Patients with osteopenia/osteoporosis at study entry experienced a marked mean increase from baseline $(+20.6,95 \% \mathrm{CI}$ : $3.1,38.1 \mathrm{mg} / \mathrm{cm}^{3} ;+2.5 \%, 95 \%$ CI: $0.5 \%, 4.6 \%$ ), as well as patients on Vitamin D supplementation $(+26.8,95 \%$ CI: $7.7,45.9 \mathrm{mg} / \mathrm{cm} 3 ;+3.4 \%, 95 \%$ CI: $1.0 \%, 5.7 \%)$. As regards $\mathrm{FN}$, increased BMD was observed, on average, in patients without TDF in the previous regimen, and those with low BMD at study entry. Both naive and experienced PLWH showed a slight and not significant percent increase in FN BMD.

Eighteen patients out of 133 (13.5\%) interrupted DTG treatment (13 adverse events, two patients' preference, one virologic failure, one clinical event, and one unknown 


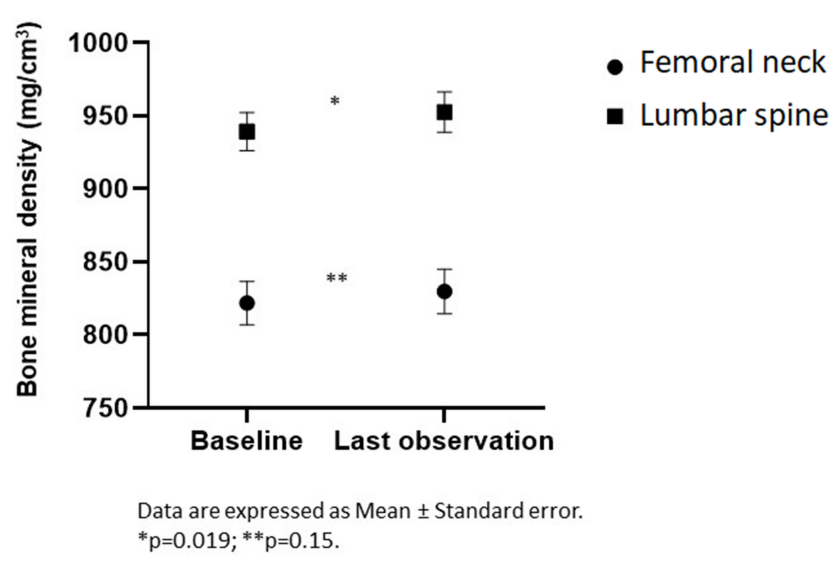

Figure I Femoral neck and lumbar spine bone mineral density at baseline and last observation.

reason) after a median time of 63 weeks (IQR: 29-93) but underwent the scheduled DXA scan. Excluding these patients from the analysis, we found similar results, although all mean estimates were higher (Table 4S).
As regards the backbone, considering 115 patients with a control DXA scan who continued the DTG-based regimen, $13(11.3 \%)$ had a switch: three on lamivudine (3TC)/ abacavir (ABC) switched to DTG plus rilpivirine (RPV) (2) or DTG alone. Two subjects on dual therapy switched to another dual (emtricitabine (FTC) to RPV and RPV to maraviroc). Seven subjects on FTC/TDF switched to FTC/ TAF (5), dual with 3TC (1), and monotherapy with DTG (1). One patient on another regimen, including FTC/TDF, replaced TDF with TAF.

Patients who started a 3TC-including regimen showed a significant increase of LS BMD at study end (17.4, 95\% CI $6.6,28.2 \mathrm{mg} / \mathrm{cm}^{3}$ ), whereas those on TDF experienced a non-statistically significant decline $(-15.0,95 \% \mathrm{CI}$ $\left.-69.3,39.3 \mathrm{mg} / \mathrm{cm}^{3}\right)$.

Analyzing experienced patients, we considered if TDF or PI exposure in the previous regimen was associated with a different change from baseline of LS and FN

Table 3 Bone Mineral Density (BMD) Change from Baseline in I 33 Subjects with Follow-Up DXA at Femoral Neck ( $n=1$ I 2) and/or Lumbar Spine $(n=128)$

\begin{tabular}{|c|c|c|c|c|c|c|c|c|}
\hline \multirow[t]{3}{*}{ Baseline Characteristics } & \multicolumn{8}{|c|}{ BMD Change from Baseline Mean $(95 \% \mathrm{Cl})$} \\
\hline & \multicolumn{4}{|l|}{$\mathbf{F N}$} & \multicolumn{4}{|l|}{ LS } \\
\hline & $\mathrm{mg} / \mathrm{cm}^{3}$ & $\mathbf{P}^{\#}$ & $\%$ & $\mathbf{P}^{\#}$ & $\mathrm{mg} / \mathrm{cm}^{3}$ & $\mathbf{P}^{\#}$ & $\%$ & $\mathbf{P}^{\#}$ \\
\hline Age $<50$ years & $-6.2(-25.5,13.1)$ & 0.10 & $-0.6(-3.1,1.9)$ & 0.08 & $19.0(1.7,36.6)^{*}$ & 0.40 & $2.2(0.2,4.2)^{*}$ & 0.38 \\
\hline Age $\geq 50$ years & $17.8(-2.4,37.9)$ & & $2.9(0.0,5.7)$ & & $9.0(-6.6,24.5)$ & & I.I $(-0.6,2.8)$ & \\
\hline Men & $8.1(-9.1,25.3)$ & 0.97 & $1.4(-1.0,3.8)$ & 0.99 & $12.2(-0.3,24.7)$ & 0.79 & $1.4(0.1,2.8)^{*}$ & 0.75 \\
\hline Women & $7.5(-19.0,34.0)$ & & $1.5(-2.1,5.0)$ & & $15.6(-11.3,42.5)$ & & $1.9(-1.3,5.1)$ & \\
\hline $\mathrm{BMI}<25.0 \mathrm{~kg} / \mathrm{m}^{2}$ & $3.7(-17.2,24.5)$ & 0.46 & $1.0(-1.9,4.0)$ & 0.61 & $-2.2(-16.4,12.0)$ & 0.0008 & $-0.1(-1.7,1.5)$ & 0.002 \\
\hline $\mathrm{BMI} \geq 25.0 \mathrm{~kg} / \mathrm{m}^{2}$ & $\mid 4.5(-2.8,3 \mid .9)$ & & $2.1(-0.2,4.4)$ & & $37.1(17.2,55.0)^{*}$ & & $4.1(2.1,6.1)^{*}$ & \\
\hline Experienced & $10.0(-6.8,26.8)$ & 0.55 & $1.7(-0.6,4.1)$ & 0.55 & $16.9(4.7,29.2)^{*}$ & 0.21 & $2.0(0.6,3.4)^{*}$ & 0.16 \\
\hline Naive & $-1.0(-23.9,21.9)$ & & $0.2(-2.7,3.1)$ & & $-1.1(-31.4,29.3)$ & & $-0.2(-3.5,3.0)$ & \\
\hline TDF in previous regimen ${ }^{\S}$ & $-11.2(-36.4,11.9)$ & 0.02 & $-1.4(-4.5,1.6)$ & 0.01 & $12.9(-5.2,3 \mid .0)$ & 0.53 & $1.6(-0.4,1.6)$ & 0.56 \\
\hline No TDF in previous regimen ${ }^{\S}$ & $28.2(6.0,50.4)^{*}$ & & $4.4(I . I, 7.8)^{*}$ & & $20.7(3.5,37.9)^{*}$ & & $2.4(0.4,4.4)^{*}$ & \\
\hline $\mathrm{PI}$ in previous regimen ${ }^{\S}$ & $-2.5(-35.0,30.0)$ & 0.24 & $0.0(-4.3,4.2)$ & 0.23 & $25.1(0.0,50.1)^{*}$ & 0.28 & $3.2(0.4,6.1)^{*}$ & 0.16 \\
\hline No $\mathrm{PI}$ in previous regimen ${ }^{\S}$ & I8.I $(-0.5,36.8)$ & & $2.9(0.1,5.6)^{*}$ & & $11.6(-0.9,24.1)$ & & $1.2(-0.2,1.6)$ & \\
\hline Normal BMD at baseline & $-1.7(-24.0,20.6)$ & 0.22 & $-0.3(-2.9,2.4)$ & 0.12 & $4.4(-10.0,18.8)$ & 0.16 & $0.4(-1.0,1.8)$ & 0.10 \\
\hline Reduced BMD at baseline & $16.0(-2.6,34.6)$ & & $2.9(0.0,5.7)^{*}$ & & $20.6(3.1,38.1)^{*}$ & & $2.5(0.5,4.6)$ & \\
\hline Vitamin D supplement & $-4.0(-27.0,19.0)$ & 0.19 & $0.6(-3.0,4.2)$ & 0.51 & $26.8(7.7,45.9)^{*}$ & 0.09 & $3.4(1.0,5.7)^{*}$ & 0.04 \\
\hline No Vitamin D supplement & $15.3(-3.0,33.7)$ & & $2.0(-0.4,4.3)$ & & $6.0(-8.4,20.3)$ & & $0.6(-0.9,6.1)$ & \\
\hline $3 \mathrm{TC} / \mathrm{ABC} / \mathrm{DTG}$ & $5.2(-9.6,20.0)$ & 0.48 & $1.2(-1.1,2.4)$ & 0.57 & I9.I (7.8, 30.5)* & 0.20 & $2.1(0.8,3.4)^{*}$ & 0.24 \\
\hline TDF/FTC/DTG & $1.8(-78.6,72.2)$ & & $0.4(-8.5,9.3)$ & & $-12.4(-70.5,45.7)$ & & $-1.0(-7.0,4.9)$ & \\
\hline Dual therapy & II.8 $(-29.4,53.0)$ & & $1.9(-3.0,6.7)$ & & $17.2(-23.2,57.5)$ & & $2.4(-2.3,7.0)$ & \\
\hline Other Therapies & $66.0(-18.2,150.2)$ & & $8.5(-2.2,19.2)$ & & $-18.3(-52.9,16.3)$ & & $-2.0(-5.6,1.6)$ & \\
\hline Never smokers & $-4.7(-29.5,4.1)$ & 0.73 & $-0.3(-3.5,2.9)$ & 0.68 & $19.7(2.9-36.6)^{*}$ & 0.06 & $2.1(0.4,3.9)^{*}$ & 0.06 \\
\hline Former smokers & $4.0(-72.6,70.6)$ & & $0.9(-6.8,8.6)$ & & $23.0(-11.6,57.6)$ & & $3.1(-1.5,7.7)$ & \\
\hline Current smokers & $11.0(-16.1 .38 .1)$ & & $2.1(-2.8,7.1)$ & & $-9.5(-28.7,9.8)$ & & $-0.9(-3.1,1.2)$ & \\
\hline
\end{tabular}

Notes: ${ }^{\#}$ Comparison between groups, analysis of variance; ${ }^{*} \mathrm{p}<0.05$ for change from baseline, paired $t$-test; ${ }^{\S}$ limited to experienced patients.

Abbreviations: BMD, bone mineral density; $\mathrm{Cl}$, confidence interval; FN, femoral neck; LS, lumbar spine; BMI, body mass index; TDF, tenofovir disoproxil fumarate; PI, protease inhibitors; 3TC, lamivudine; ABC, abacavir; DTG, dolutegravir; FTC, emtricitabine; smoking status was only reported in 88 patients. 
BMD: patients exposed to PI but not to TDF had higher BMD change from baseline (Table 3 ).

At baseline, mean estimated Glomerular filtration rate (eGFR) was $90.8 \pm 21.4 \mathrm{~mL} / \mathrm{min}$, significantly different between experienced and naïve patients $(87.2 \pm 20.6$ vs $102.2 \pm 20.1 \mathrm{~mL} / \mathrm{min}, \mathrm{p}=0.0001$ ) as expected. Over time, we observed a decline: at the first follow-up visit, mean eGFR was $80.6 \pm 20.6$ and $86.8 \pm 18.9$ in experienced and naïve subjects. After that, the eGFR remained substantially unchanged. At the time of the last BMD scan, the change from baseline of eGFR $(-7.7,95 \% \mathrm{CI}:-10.4,-5.1 \mathrm{~mL} / \mathrm{min})$ was not associated with $\mathrm{FN}$ and LS variations (Pearson $\mathrm{r}=$ $-0.11, p=0.22$ for $F N$, Pearson $r=-0.03, p=0.71$ for LS).

\section{Multivariate Analysis}

In a multivariable model, including age, sex, BMI, naïve status, vitamin D supplementation, osteopenia/osteoporosis at baseline, and weeks to follow-up scan, patients with osteopenia/osteoporosis, as well as experienced subjects, still had a significant modification of LS BMD (both absolute and percentage).

We found that positive LS change from baseline was confirmed associated with age $<50$ years, male sex, BMI $\geq 25.0 \mathrm{~kg} / \mathrm{m}^{2}$, osteopenia or osteoporosis at baseline, and vitamin D supplement. Comparing classes, LS change from baseline was significantly higher in subjects with $\mathrm{BMI} \geq 25.0 \mathrm{Kg} / \mathrm{m}^{2}$ than in those with $\mathrm{BMI}<25.0 \mathrm{Kg} /$ $\mathrm{m}^{2}$ (both absolute and percentage).

Limiting the analysis to experienced subjects, we found that, including all previously cited factors but naïve status, and including previous TDF and PI exposure in the model at the same time, patients exposed to PI in the previous regimen significantly increased LS BMD.

\section{Smoking Status}

Since the information on smoking status was unavailable in $27.5 \%$ of subjects at enrolment and $33.8 \%$ of those with follow-up scans, we did not include this variable in the main analysis. However, its role in bone density is well established. Thus, we analyzed change from baseline in patients with information. Never smokers showed, at univariate analysis, a significant increase of LS BMD (Table 3), whereas the analysis of variance among smoking status groups was of borderline significance. In the multivariate analysis, we run the model on the subset of 88 patients with known smoking status, finding a significant difference in the overall analysis of variance due to the comparison between current and never smokers $(\mathrm{p}=0.0008)$. In contrast, no difference was observed between former and never smokers.

\section{Sensitivity Analysis}

All previous findings were confirmed, running the analyses with the exclusion of patients who interrupted DTG treatment. We also reran the same models excluding patients who switched from TDF to TAF, to assess if these findings were driven by this switch: we found similar results.

The same analysis was also run, including previous use of intravenous drugs (IDU): BMD change from baseline was not significantly different between groups (Table 5S). However, LS BMD showed a higher change from baseline in subjects without IDU history (15.1, 95\% CI 2.6, $27.6 \mathrm{mg} / \mathrm{cm}^{3}$ vs $\left.0.3,95 \% \mathrm{CI}-30.2,30.8\right)$, confirmed in the multivariate analysis.

Lastly, regimen type (dual, TDF/FTC+DTG, 3TC/ABC +DTG, and other) were included in the equation model: LS BMD change from baseline was significant for 3TC/ ABC+DTG (Table 3).

\section{Discussion}

In the past two decades, osteoporosis emerged as significant comorbidity in PLWH. ${ }^{4,11,35}$ In addition to traditional risk factors for fracture, specific factors related to HIV infection are also likely to contribute, including antiretroviral therapy. ${ }^{1}$ More specifically, HIV-infected individuals have both traditional and specific HIV-related risk factors for low BMD and fracture. ${ }^{3}$ In the former category, tobacco use, alcohol abuse, hypogonadism, exposure to trauma, and low BMI may be more prevalent in HIVinfected individuals, particularly intravenous drug users. $^{36}$ Risk factors specific to HIV infection include ART, chronic inflammation, and comorbidities.

Our study is, to our knowledge, the first prospective evaluation of BMD of PLWH after starting dolutegravir in a "real-life" setting, including naïve and antiretroviral (ARV) experienced patients on DTG-based regimens. After a median follow-up of 102 weeks, we found in experienced PLWH a not significant increase in BMD at FN and a statistically significant increase at LS. Interestingly, we also showed a not statistically significant decrease in both sites in naïve PLWH.

Among antiretroviral classes, INSTI showed a good bone safety profile in both naïve and ARV-experienced PLWH. More specifically, raltegravir has proved to be associated with less bone loss in respect of both darunavir/ritonavir (r) or atazanavir/r when combined with TDF/ 
FTC in a randomized clinical trial enrolling 328 naïve PLWH. ${ }^{26}$ Furthermore, raltegravir associated with daruna$\mathrm{vir} / \mathrm{r}$ in a dual therapy strategy has been associated with an increase in whole-body BMD over 48 weeks that was significantly higher when compared to darunavir/ritonavir $(\mathrm{DRV} / \mathrm{r})+\mathrm{TDF} / \mathrm{FTC}^{19}$ in naïve PLWH. The same strategy was tested in the bone sub-study of the randomized clinical NEAT001/ANRS143 trial enrolling 146 naïve PLWH, showing a significantly lower BMD loss at both spine and hip in the raltegravir + darunavir/ $\mathrm{r}$ arm compared to the triple arm including TDF. ${ }^{37}$ Raltegravir, in combination with a boosted PI, has also been associated with a significant increase in BMD at both spine and hip at weeks 24 and 48, after switching from a triple therapy including TDF, in virologically suppressed PLWH with low BMD (<-1.0 T-score) ${ }^{38}$ In the OsteoTDF study, hip BMD improved by $2.1 \%(95 \% \mathrm{CI}-0.6$ to 4.7$)(\mathrm{P}=0.043)$ in PLWH switching to abacavir compared to $0.7 \%$ (95\% $\mathrm{CI}-0.9$ to 2.4$)(\mathrm{P}=0.372)$ in the tenofovir group. ${ }^{39}$

Elvitegravir has also proved safe on bone, especially when combined with cobicistat/TAF/FTC in comparison with cobicistat/TDF/FTC, it was associated with a significantly lower decline in BMD in naive patients at both spine and hip after 144 weeks, in 2 double-blind, randomized clinical trials. ${ }^{40}$ Significant improvements in hip and spine BMD were also evidenced for those virologically suppressed patients switched to elvitegravir/cobicistat/TAF/FTC versus continuing TDF, over week 96 in a randomized clinical trial. ${ }^{41}$

Dolutegravir impact on bone has been evaluated in the GS-US-380-1489 study on antiretroviral therapy-naive patients, in which similar small decrease in hip and lumbar spine BMD were evidenced both in patients on bictegravir (BIC)/TAF/FTC and DTG/ABC/3TC. ${ }^{42}$ Similar and small increases from baseline to week 48 were seen in hip and lumbar spine $\mathrm{BMD}$, after switching from $\mathrm{DTG} / \mathrm{ABC} / 3 \mathrm{TC}$ to $\mathrm{BIC} / \mathrm{TAF} / \mathrm{FTC}$ or continuing $\mathrm{DTG} / \mathrm{ABC} / 3 \mathrm{TC}$ in a randomized clinical trial conducted in virologically suppressed PLWH. ${ }^{43}$ Dolutegravir impact on bone was also evaluated in a sub-study of two trials, comparing the switch to dual therapy, including dolutegravir plus rilpivirine vs remaining on a triple therapy including TDF. Patients switching to DTG plus rilpivirine showed a significantly higher percent increase compared to those remaining in the triple regimen, at both spine and hip at week $48 .^{27}$ More recently, an Italian pilot study on 16 virologically suppressed PLWH showed a significant increase in spine BMD after switching from triple therapy to dolutegravir plus lamivudine. ${ }^{28}$ Lastly, the randomized DOMONO trial studied bone health after simplifying cART to DTG monotherapy. ${ }^{44}$ Although this simplification strategy was demonstrated inferior to cART, it showed a positive effect on FN and LS bone density, adding to the accumulating evidence about the bone safety of dual therapy regimens.

Taken together, the studies on the bone impact of INSTI are reassuring in evidencing a lower bone loss when compared to other drug classes, even if associated with TDF.

In particular, DTG has been associated with an increase in BMD in virologically suppressed patients in dual therapy regimens ${ }^{27,28}$ and monotherapy ${ }^{44}$ and with a not significant decrease when combined with $\mathrm{ABC} / 3 \mathrm{TC}$, with a similar effect if compared to $\mathrm{BIC} / \mathrm{TAF} / \mathrm{FTC}$ in naive patients. ${ }^{42,43}$

Vitamin D supplementation is known to increase BMD in the general population. HIV-infected patients are at risk of vitamin D deficiency, especially in those receiving protease inhibitors. ${ }^{45}$ Determination of vitamin D status should be part of the management of PLWH, and appropriate supplementation is suggested. ${ }^{46}$

Our results confirm in "real life" the results of clinical studies, suggesting that dolutegravir is associated with a good bone safety profile, especially when combined with $\mathrm{ABC} / 3 \mathrm{TC}$. Interestingly, these results are not only evidenced in the overall cohort but also patients with osteopenia/osteoporosis, with an elevated risk of fracture, who showed a higher BMD increase during follow-up when compared with patients with normal BMD.

Some limitations of the present study need to be discussed. Firstly, this analysis was conducted on a subset of patients, referring to centers where DXA scan was available and routinely carried out; thus, these centers cannot be considered representative of all Italian Infectious Diseases Clinics. Secondly, considering that patients were selected only if they consented to undergo a DXA scan, it is likely that older patients and those with higher health consciousness gave their consent. However, as regards known patients' characteristics, we did not observe significant differences between those who consented and those who declined the DXA examination, although the possibility of unknown confounders cannot be excluded. Thirdly, we could not account for some lifestyle habits (alcohol intake and physical activity, both occupational and leisure) and menopausal status in women. However, smoking habits, history of drug 
abuse were accounted for, and patients with hypogonadism and comorbidities requiring glucocorticoids were excluded from the study. Lastly, we have to acknowledge the small sample size, the lack of a control group of PLWH not receiving DTG containing regimens, and the potential self-selection of patients with osteopenia/osteoporosis could represent biases for our analysis.

In conclusion, our data suggest that dolutegravircontaining regimens represent an excellent option to reduce the impact of antiretroviral therapy on bone, especially in patients with low BMD, to reduce bone loss and fracture risk.

\section{Abbreviations}

DTG, dolutegravir; BDM, bone mineral density; AE, adverse events; FN, femoral neck; LS, lumbar spine; BL, baseline; cART, combination antiretroviral therapy; PLWH, people living with HIV; BMI, body mass index; $\mathrm{HCV}$, hepatitis $\mathrm{C}$ virus; HBV, hepatitis $\mathrm{B}$ virus; TDF, tenofovir disoproxil fumarate; TAF, tenofovir alafenamide; MDRD, Modification of Diet in Renal Diseases; DXA, dual-energy X-ray absorptiometry; SD, standard deviation; IQR, interquartile range; CI, confidence intervals; ITT, intention-to-treat; $\mathrm{CDC}$, Centers for Disease Control and Prevention; PI, protease inhibitor; 3TC, lamivudine; ABC, abacavir; RPV, rilpivirine; FTC, emtricitabine BIC, bictegravir; IDU, intravenous drugs user; eGFR, estimated Glomerular filtration rate; ARV, antiretroviral; INSTI, integrase inhibitors; DRV/r, darunavir/ritonavir.

\section{Acknowledgments}

The authors thank all the patients and physicians who were involved in this study. The Coordinamento Italiano Studio Allergie e Infezione da HIV (C.I.S.A.I.) comprises the following members: Coordinators: P. Bonfanti (Monza), A. di Biagio (Genova). Data Manager: E. Ricci. Participating center: E. Sarchi, G. Chichino, C. Bolla, A. Parisini, E. Blasi Vacca (Alessandria); C. Bellacosa, G. Angarano (Bari); L. Calza (Bologna); B. Menzaghi, M. Farinazzo (Busto Arsizio); G. Angioni (Cagliari); M. Gussio, B.M. Celesia (Catania); K. Falasca (Chieti); A. Mastroianni, G. Guadagnino (Cosenza); F. Vichi, E. Salomoni (Firenze); C. Martinelli (Firenze); A. Di Biagio, L. Nicolini (Genova); P. Bonfanti, C. Molteni (Lecco); G.F. Pellicanò, A. Nunnari (Messina); L. Valsecchi, L. Cordier, S. Parisini, G. Rizzardini (Milano); S. Rusconi, F. Conti (Milano); A. Bandera, L. Taramasso, A. Gori (Milano); D. Motta, M. Puoti (Milano); N. Squillace, G.M. Migliorino (Monza); P. Maggi, S. Martini
(Napoli); A. Cascio, M. Trizzino (Palermo); R. Gulminetti (Pavia); G.V. De Socio, D. Francisci (Perugia); D. Cibelli, G. Parruti (Pescara); G. Cenderello, C. Dentone (Sanremo); G. Madeddu, A. De Vito, M.S. Mameli (Sassari); G. Orofino, M. Guastavigna (Torino).

\section{Author Contributions}

$\mathrm{PB}, \mathrm{ER}$, and GM contributed the study conception and design, analysis and interpretation of the data and drafting of the article; ADV contributed in analysis and interpretation of the data, and drafting the article; $\mathrm{BM}, \mathrm{GO}, \mathrm{NS}$, CM, GVLDS, ES, BMC, CD, and VC, made substantial contributions in the acquisition and interpretation of data, and they critically reviewed the manuscript. All authors agreed to submit to the current journal, gave final approval of the version to be published, and agree to be accountable for all aspects of the work.

\section{Funding}

The study has received unrestricted funding from ViiV HealthCare Srl.

\section{Disclosure}

PB has received consultancy and/or speakers' fees from Gilead Sciences, Janssen, Merck Sharp \& Dohme and ViiV. GVDS has received travel grants, and speaker's honoraria from Gilead, ViiV Healthcare, Janssen-Cilag and MSD. ER has acted as statistician consultation for ViiV. BMC received grants, travel grants and speaker's honoraria from Abbvie, Bristol-Myers Squibb, Gilead, ViiV, Janssen-Cilag and Merck Sharp \& Dohme, was advisor for Bristol-Myers Squibb, Gilead, ViiV, JanssenCilag and Merck Sharp \& Dohme and received fellowship from ViiV and Gilead. GM has acted as advisor for Gilead Sciences, Janssen and Merck Sharp and Dohme and ViiV Healthcare and has received speakers' honoraria from Gilead Sciences, Merck Sharp and Dohme, Janssen and ViiV Healthcare. NS received grants for consultancy from ViiV Healthcare, and grants for participations at meetings from Gilead and Janssen. ADV, BM, ES, GO, VC and CD report no conflicts of interest in this work.

\section{References}

1. Compston J. HIV infection and osteoporosis. Bonekey Rep. 2015;4:636. doi:10.1038/bonekey.2015.3

2. Shiau S, Broun EC, Arpadi SM, Yin MT. Incident fractures in HIV-infected individuals: a systematic review and meta-analysis. AIDS. 2013;27(12):1949-1957. doi:10.1097/QAD.0b013e328361d241 
3. Premaor MO, Compston JE. The hidden burden of fractures in people living with HIV. JBMR Plus. 2018;2(5):247-256. doi:10.1002/ jbm4.10055

4. Brown TT, Qaqish RB. Antiretroviral therapy and the prevalence of osteopenia and osteoporosis: A meta-analytic review. AIDS. 2006;20 (17):2165-2174. doi:10.1097/QAD.0b013e32801022eb

5. Ofotokun I, Titanji K, Vunnava A, et al. Antiretroviral therapy induces a rapid increase in bone resorption that is positively associated with the magnitude of immune reconstitution in HIV infection. AIDS. 2016;30(3):405-414. doi:10.1097/QAD.0000000000000918

6. Kung YY, Felge U, Sarosi I, et al. Activated T cells regulate bone loss and joint destruction in adjuvant arthritis through osteoprotegerin ligand. Nature. 1999;402(6759):304-309. doi:10.1038/46303

7. Compston J. HIV infection and bone disease. J Intern Med. 2016;280 (4):350-358. doi:10.1111/joim. 12520

8. Hidron AI, Hill B, Guest JL, Rimland D. Risk factors for vitamin $\mathrm{D}$ deficiency among veterans with and without HIV infection. PLoS One. 2015;10(4):e0124168. doi:10.1371/journal.pone.0124168

9. Dao CN, Patel P, Overton ET, et al. Low vitamin D among HIV-infected adults: prevalence of and risk factors for low vitamin D levels in a cohort of HIV-infected adults and comparison to prevalence among adults in the us general population. Clin Infect Dis. 2011;52(3):396-405. doi:10.1093/cid/ciq158

10. Yin M, Stein E. The effect of antiretrovirals on vitamin D. Clin Infect Dis. 2011;52(3):406-408. doi:10.1093/cid/ciq169

11. Madeddu G, Spanu A, Solinas P, et al. Bone mass loss and vitamin $\mathrm{D}$ metabolism impairment in HIV patients receiving highly active antiretroviral therapy. QJ Nucl Med Mol Imaging. 2004;48(1):39-48.

12. Pinzone MR, Di Rosa M, Celesia BM, et al. LPS and HIV gp120 modulate monocyte/macrophage CYP27B1 and CYP24A1 expression leading to vitamin D consumption and hypovitaminosis D in HIV-infected individuals. Eur Rev Med Pharmacol Sci. 2013;17(14):1938-1950.

13. Madeddu G, Spanu A, Solinas P, et al. Different impact of NNRTI and PI-including HAART on bone mineral density loss in HIV-infected patients. Eur Rev Med Pharmacol Sci. 2015;19 (23):4576-4589.

14. Rgen Stellbrink H-J, Orkin C, Arribas JR, et al. Comparison of changes in bone density and turnover with abacavir-lamivudine versus tenofovir-emtricitabine in HIV-infected adults: 48-week results from the ASSERT study. Clin Infect Dis. 2010;51(8):963-972. doi: $10.1086 / 656417$

15. Hileman CO, Overton ET, McComsey GA. Vitamin D and bone loss in HIV. Curr Opin HIV AIDS. 2016;11(3):277-284. doi:10.1097/ COH.0000000000000272

16. Sax PE, Wohl D, Yin MT, et al. Tenofovir alafenamide versus tenofovir disoproxil fumarate, coformulated with elvitegravir, cobicistat, and emtricitabine, for initial treatment of HIV-1 infection: two randomised, double-blind, Phase 3, non-inferiority trials. Lancet. 2015;385(9987):2606-2615. doi:10.1016/S0140-6736(15)60616-X

17. Mills A, Arribas JR, Andrade-Villanueva J, et al. Switching from tenofovir disoproxil fumarate to tenofovir alafenamide in antiretroviral regimens for virologically suppressed adults with HIV-1 infection: a randomised, active-controlled, multicentre, open-label, phase 3, non-inferiority study. Lancet Infect Dis. 2016;16(1):43-52. doi:10.1016/S1473-3099(15)00348-5

18. Post FA, Tebas P, Clarke A, et al. Switching to tenofovir alafenamide, coformulated with elvitegravir, cobicistat, and emtricitabine, in HIV-infected adults with renal impairment: 96-week results from a single-arm, multicenter, open-label phase 3 study. J Acquir Immune Defic Syndr. 2017;71(5):530-537. doi:10.1097/QAI.0000000000001186

19. Bedimo RJ, Drechsler H, Jain M, et al. The RADAR study: week 48 safety and efficacy of RAltegravir combined with boosted DARunavir compared to tenofovir/emtricitabine combined with boosted darunavir in antiretroviral-naive patients. Impact on bone health. PLoS One. 2014;9(8):e106221. doi:10.1371/journal. pone. 0106221
20. Brown TT, Moser C, Currier JS, et al. Changes in bone mineral density after initiation of antiretroviral treatment with Tenofovir Disoproxil Fumarate/Emtricitabine Plus Atazanavir/ Ritonavir, Darunavir/ Ritonavir,or Raltegravir. J Infect Dis. 2015;212 (8):1241-1249. doi:10.1093/infdis/jiv194

21. Borghetti A, Baldin G, Capetti A, et al. Efficacy and tolerability of dolutegravir and two nucleos(t)ide reverse transcriptase inhibitors in HIV-1-positive, virologically suppressed patients. AIDS. 2017;31 (3):457-459. doi:10.1097/QAD.0000000000001357

22. Rossetti B, Baldin G, Sterrantino G, et al. Efficacy and safety of dolutegravir-based regimens in advanced HIV-infected naïve patients: results from a multicenter cohort study. Antiviral Res. 2019;169. doi:10.1016/j.antiviral.2019.104552

23. Bagella P, Squillace N, Ricci E, et al. Lipid profile improvement in virologically suppressed HIV-1-infected patients switched to dolutegravir/abacavir/lamivudine: data from the SCOLTA project. Infect Drug Resist. 2019;12:1385-1391. doi:10.2147/IDR.S203813

24. Trottier B, Lake JE, Logue K, et al. Dolutegravir/abacavir/lamivudine versus current ART in virally suppressed patients (STRIIVING): a 48-week, randomized, non-inferiority, open-label, Phase IIIb study. Antivir Ther. 2017;22(4):295-305. doi:10.3851/IMP3166

25. Gatell JM, Assoumou L, Moyle G, et al. Switching from a ritonavir-boosted protease inhibitor to a dolutegravir-based regimen for maintenance of HIV viral suppression in patients with high cardiovascular risk. AIDS. 2017;31(18):2503-2514. doi:10.1097/ QAD.0000000000001675

26. Tebas P, Kumar P, Hicks C, et al. Greater change in bone turnover markers for efavirenz/emtricitabine/tenofovir disoproxil fumarate versus dolutegravir $\mathrm{R}$ abacavir/lamivudine in antiretroviral therapy-naive adults over 144 weeks. AIDS. 2015;29 (18):2459-2464. doi:10.1097/QAD.0000000000000863

27. McComsey GA, Lupo S, Parks D, et al. Switch from tenofovir disoproxil fumarate combination to dolutegravir with rilpivirine improves parameters of bone health. AIDS. 2018;32(4):477-485. doi:10.1097/QAD.0000000000001725

28. Ciccullo A, Avino AD, Lassandro AP, et al. Changes in bone mineral density in HIV-positive, virologically suppressed patients switching to lamivudine/dolutegravir dual therapy: preliminary results from clinical practice. Infez Med. 2018;26(4):336-340.

29. DAIDS. Division of aids table for grading the severity of adult and pediatric adverse events division of aids table for grading the severity of adult and pediatric adverse events. National Institute of Allergy and Infectious Diseases; 2017. Available from: https://rsc.niaid.nih.gov/ sites/default/files/daidsgradingcorrectedv21.pdf. Accessed July 3, 2020.

30. Madeddu G, De Socio GVL, Ricci E, et al. Muscle symptoms and creatine phosphokinase elevations in patients receiving raltegravir in clinical practice: results from the SCOLTA project long-term surveillance. Int $J$ Antimicrob Agents. 2015;45(3):289-294. doi:10.1016/j.ijantimicag.2014.10.013

31. Bonfanti P, Martinelli C, Ricci E, et al. An Italian approach to postmarketing monitoring: preliminary results from the SCOLTA (Surveillance Cohort Long-term Toxicity Antiretrovirals) project on the safety of lopinavir/ritonavir. J Acquir Immune Defic Syndr. 2005;39(3):317-320. doi:10.1097/01.qai.0000164248.56722.3c

32. DHHS Panel on antiretroviral guidelines for adults and adolescents. Guidelines for the use of antiretroviral agents in adults and adolescents Living with HIV developed. Department of Health and Human Services. doi:10.1108/14630010710742455

33. Wilson KE Practical consideration when replacing a DXA system. Available from: https://hologiced.com/wp-content/uploads/2018/06/ Wilson-KE.-Practical-Considerations-When-Replacing-a-DXASystem.pdf. Accessed July 3, 2020.

34. Pedrazzoni M, Girasole G, Bertoldo F, et al. Definition of a population-specific DXA reference standard in Italian women: the Densitometric Italian Normative Study (DINS). Osteoporos Int. 2003;14(12):978-982. doi:10.1007/s00198-003-1521-1 
35. Kanis JA. Assessment of fracture risk and its application to screening for postmenopausal osteoporosis. Report of a WHO Study Group. World Health Organ - Tech Rep Ser. 1994;4(6):368-381.

36. De Socio GV, Maggi P, Ricci E, et al. Smoking habits in human immunodeficiency virus-infected people from Italy: a cross-sectional analysis of the STOPSHIV cohort. AIDS Res Hum Retroviruses. 2019;36(1):19-26. doi:10.1089/aid.2019.0115

37. Bernardino JI, Mocroft A, Mallon PW, et al. Bone mineral density and inflammatory and bone biomarkers after darunavir-ritonavir combined with either raltegravir or tenofovir-emtricitabine in antiretroviral-naive adults with HIV-1: a substudy of the NEAT001/ ANRS143 randomised trial. Lancet HIV. 2015;2(11):464-473. doi:10.1016/S2352-3018(15)00181-2

38. Bloch M, Tong W, Hoy J, et al. Switch from tenofovir to raltegravir increases low bone mineral density and decreases markers of bone turnover over 48 weeks. HIV Med. 2014;15(6):373-380. doi:10.1111/hiv.12123

39. Nia Negredo E, Domingo P, Pérez-Álvarez N, et al. Improvement in bone mineral density after switching from tenofovir to abacavir in HIV-1-infected patients with low bone mineral density: two-centre randomized pilot study (OsteoTDF study). J Antimicrob Chemother. 2014;69:3368-3371. doi:10.1093/jac/dku300

40. Arribas JR, Thompson M, Sax PE, et al. Brief report: randomized, double-blind comparison of tenofovir alafenamide (TAF) vs Tenofovir Disoproxil Fumarate (TDF), Each Coformulated with Elvitegravir, Cobicistat, and Emtricitabine (E/C/F) for Initial HIV-1 treatment: week 144 results. J Acquir Immune Defic Syndr. 2017;75 (2):211-218. doi:10.1097/QAI.0000000000001350
41. DeJesus E, Haas B, Segal-Mauer S, et al. Superior efficacy and improved renal and bone safety after switching from a tenofovir disoproxil fumarate (TDF)- to a Tenofovir Alafenamide (TAF)-based Regimen Through 96 Weeks of Treatment. AIDS Res Hum Retroviruses. 2018;34(4):337-342. doi:10.1089/aid.2017.0203

42. Gallant J, Lazzarin A, Mills A, et al. Bictegravir, emtricitabine, and tenofovir alafenamide versus dolutegravir, abacavir, and lamivudine for initial treatment of HIV-1 infection (GS-US-380-1489): a double-blind, multicentre, phase 3, randomised controlled non-inferiority trial. Lancet. 2017;390(10107):2063-2072. doi:10.1016/S0140-6736(17)32299-7

43. Molina JM, Ward D, Brar I, et al. Switching to fixed-dose bictegravir, emtricitabine, and tenofovir alafenamide from dolutegravir plus abacavir and lamivudine in virologically suppressed adults with HIV-1: 48 week results of a randomised, double-blind, multicentre, active-controlled, phas. Lancet HIV. 2018;5(7):e357-e365. doi:10.1016/S2352-3018(18)30092-4

44. Wijting IEA, Rokx C, Zillikens MC, et al. Changes in renal, bone, lipid, and inflammation markers in HIV-1 patients after combination antiretroviral therapy simplification to dolutegravir monotherapy. Int J STD AIDS. 2019;30(11):1042-1048. doi:10.1177/0956462419848962

45. Vescini F, Cozzi-Lepri A, Borderi M, et al. Prevalence of hypovitaminosis $\mathrm{D}$ and factors associated with vitamin $\mathrm{D}$ deficiency and morbidity among HIV-infected patients enrolled in a large Italian cohort. J Acquir Immune Defic Syndr. 2011;58(2):163-172. doi:10.1097/QAI.0b013e31822e57e9

46. Brown TT, Hoy J, Borderi M, et al. Recommendations for evaluation and management of bone disease in HIV. Clin Infect Dis. 2015;60 (8):1242-1251. doi:10.1093/cid/civ010
Infection and Drug Resistance

\section{Publish your work in this journal}

Infection and Drug Resistance is an international, peer-reviewed openaccess journal that focuses on the optimal treatment of infection (bacterial, fungal and viral) and the development and institution of preventive strategies to minimize the development and spread of resistance. The journal is specifically concerned with the epidemiology of antibiotic resistance and the mechanisms of resistance development and diffusion in both hospitals and the community. The manuscript management system is completely online and includes a very quick and fair peerreview system, which is all easy to use. Visit http://www.dovepress.com/ testimonials.php to read real quotes from published authors. 